\title{
Validation of suitable reference genes for quantitative polymerase chain reaction analysis in rabbit bone marrow mesenchymal stem cell differentiation
}

\author{
HECHENG MA ${ }^{1}$, QIWEI YANG ${ }^{2}$, DONGSONG LI ${ }^{1}$ and JIANGUO LIU ${ }^{1}$ \\ ${ }^{1}$ Department of Orthopedics, The First Hospital of Jilin University, Jilin University, Changchun, Jilin 130021; \\ ${ }^{2}$ Central Laboratory, Jilin University, China-Japan Union Hospital, Changchun, Jilin 130033, P.R. China
}

Received May 2, 2014; Accepted January 15, 2015

DOI: $10.3892 / \mathrm{mmr} .2015 .3776$

\begin{abstract}
Bone marrow mesenchymal stem cells (BMSCs) are considered as multipotent cells, representing a multilineage potential to differentiate into mesodermal lineages of mesenchymal tissues, including cartilage, bone, fat, muscle and tendon. Tissue engineering in BMSCs has made great advances in the regeneration of cartilage and bone defects. To uncover the mechanisms of the multipotent differentiation process, the molecular changes in gene expression profiles during chondrogenic and osteogenic differentiation need to be evaluated with reliable, accurate, fast and sensitive methods. Reverse transcription-quantitative polymerase chain reaction is a commonly used technology for analyzing gene expression, depending on an appropriate reference gene to normalize the errors. The commonly used reference genes vary, and no ideal and universal reference genes suitable for all conditions exist; therefore validation of the stability of gene expression is required. In the present study, three common statistical algorithms, geNorm, Normfinder and BestKeeper, were used to identify the expression stability of 12 genes, and the target differentiation markers during the differentiation of BMSCs were evaluated accurately. Our results demonstrated that YWHAZ, PPIA and GAPDH were suitable as reference genes for chondrogenic differentiation, while RPL13a allowed an efficient normalization expression value of interest genes for osteogenic differentiation of BMSCs. By contrast, the most unstable reference genes were 18s rRNA, B2M and HPRT1 in all studies, and these should be avoided when investigating the differentiation of BMSCs. Our results demonstrate validation
\end{abstract}

Correspondence to: Professor Jianguo Liu, Department of Orthopedics, The First Hospital of Jilin University, Jilin University, 71 Xinmin Street, Changchun, Jilin 130021, P.R. China E-mail:yopo2007@qq.com

Key words: bone marrow mesenchymal stem cells, osteogenic and chondrogenic differentiation, quantitative polymerase chain reaction, reference genes of the appropriate reference genes for accurate gene expression in chondrogenic and osteogenic differentiation of BMSCs.

\section{Introduction}

Bone marrow contains a rare population of mesenchymal stem cells (BMSCs), derived from the mesodermal layer during embryologic development, and represents a multilineage potential to differentiate into mesodermal lineages of mesenchymal tissues, including cartilage, bone, fat, muscle and tendon $(1,2)$. Previous studies have demonstrated that BMSCs even differentiate into tissues in various germ layers beyond the normal embryonic limitation (mesodermal layer). For example, BMSCs have the potential to differentiate into neuronal (ectoderm) (3), pancreatic (4) and hepatic (entoderm) (5) tissue.

In the last decades, the isolation and culture of BMSCs has been well illustrated (6), and they can be further purified by their cell surface expression as specific markers (7). Moreover, the particular techniques to induce BMSC differentiation into chondrocytes (8), osteocytes (9), adipocytes (10) and myocytes (11) have been described. BMSCs provide excellent candidates for cell-based tissue engineering due to their self-renewal and multipotent differentiation capacity (12).

Mesenchymal stem cells derived from the bone marrow differentiate along tissue-specific lineages and microenvironments when transplanted to an organ defect. Guan et al developed a specific peptidomimetic ligand to direct BMSCs to the defect bone surface and induced bone regeneration and bone strength (9). Additionally, cartilage defects have been enhanced and regenerated by the transplantation of BMSCs in a rabbit model (13) and clinical patients (14). Furthermore, Horwitz et al demonstrated that transplantation of BMSCs in children with osteogenesis and imperfecta allogeneic increased bone marrow density (15).

There have been great achievements in tissue engineering regeneration in BMSCs, and the molecular changes in gene expression profiles during proliferation, differentiation and redifferentiation are crucial to explain the mechanisms of the multipotent differentiation process, which depends on reliable, accurate, fast and sensitive methods. As a powerful technique to rapidly quantify gene expression, 
reverse transcription-quantitative polymerase chain reaction (RT-qPCR) is likely to play a vital role in deciphering the cellular and molecular properties of BMSC differentiation with high sensitivity. As a quantitative analysis, however, RT-qPCR depends on an appropriate reference gene to normalize cell number, RNA extraction, sample-to-sample variations, reverse transcription and amplification efficiency differences (16). The appropriate reference genes are required to be constitutively expressed in the cells or tissue for the various investigations, generally undergoing basic cellular functions and being expressed at abundant levels (17). However, evidence has revealed that the commonly used reference gene expression levels, although occasionally constant in certain cell types and experimental conditions, varied considerably in different tissue, BMSC proliferation and differentiation (18). There are still no ideal and universal reference genes suitable for all experimental conditions (17). As a result, the presumed stability of the reference gene expression must be validated under the particular experimental conditions being investigated, although validation is lacking in most current research (19). Much research has been carried out to identify a suitable reference gene for BMSCs; however, no comprehensive conclusion has been drawn due to the few reference genes and experimental conditions studied (20).

In the present study, we selected a series of well-known and commonly used reference genes, and validated the gene expression during BMSC differentiation to osteogenic and chondrogenic lineages.

\section{Materials and methods}

Isolation and culture of rabbit BMSCs. The present study was approved by the Ethics Committee of the First Hospital of Jilin University (Changchun, Jilin). Rabbit BMSCs were isolated and cultured according to Haynesworth et al (21). Briefly, 4-week-old New Zealand rabbits from the animal center of Jilin University, China were anesthetized and the bone marrow was washed out by a needle with low-glucose Dulbecco's modified Eagle's medium (DMEM) containing 10\% fetal bovine serum (FBS; Gibco Life Technologies, Carlsbad, CA, USA), penicillin $(50 \mathrm{U} / \mathrm{ml})$ and streptomycin $(50 \mathrm{U} / \mathrm{ml})$. Cells were incubated at $37^{\circ} \mathrm{C}$ with $5 \% \mathrm{CO}_{2}$. Non-adherent cells were removed on day 3 at the first change of medium. When the adherent mesenchymal stem cells became confluent, the BMSCs were digested, detached and continuously passaged for subculture.

Chondrogenic differentiation. The BMSCs were seeded in six-well plates at 100,000 cells per well in $2 \mathrm{ml}$ chondrogenic medium. Chondrogenic medium was composed of high-glucose DMEM with 10\% FBS, 10 ng/ml transforming growth factor $\beta-1$ (Peprotech, Inc., Rocky Hill, NJ, USA) and $50 \mathrm{mM}$ ascorbate-2-phosphate (Sigma-Aldrich, St. Louis, MO, USA). The differentiation medium was replaced twice a week for 14 days.

Osteogenic differentiation. Generally, the BMSCs were seeded in six-well plates at 100,000 cells per well in $2 \mathrm{ml}$ osteogenic medium, composed of high-glucose DMEM with 10\% FBS, 10 nM dexamethasone (Sigma-Aldrich),
$50 \mathrm{mM}$ ascorbate-2-phosphate (Sigma-Aldrich) and $2 \mathrm{mM}$ $\beta$-glycerophosphate (Sigma-Aldrich). The differentiation medium was replaced twice a week for 14 days.

Selection of potential reference genes and design of reference primers. In the present study, 12 candidate reference genes were selected for investigation in accordance with a previous study (16). Primer pairs of the 12 candidate reference genes were designed by the Primer Premier 5.0 software (Premier Biosoft, Palo Alto, CA, USA). The high scored primer pairs were checked using the Primer-BLAST tool from NCBI to ensure the specificity of amplification. The primer pair sequences of the 12 candidate reference genes are listed in Table I. Prior to the expression analysis of the reference genes, the specificity of the primers was evaluated by the melting curve method following amplification with qPCR, where a single high and thin peak was considered as good specificity. Furthermore, the end-products were electrophoresed with $1.5 \%$ agarose gel and stained with ethidium bromide. The single product appeared at the expected site, suggesting good specificity.

Total RNA isolation and cDNA synthesis. On days 1, 7 and 14 of BMSC chondrogenic and osteogenic differentiation, cells were washed twice in phosphate-buffered saline and total RNA was immediately isolated using TRIzol reagent (Invitrogen Life Technologies, Carlsbad, CA, USA) according to the manufacturer's instructions. RNA concentration was evaluated with a microplate reader (Tecan, Männedorf, Switzerland) and the 260/280 ratio was between 1.8 and 2.0, which indicates pure RNA that is suitable for qPCR analysis. Then $2 \mu \mathrm{g}$ isolated total RNA was reverse transcribed to cDNA according to the manufacturer's instructions of the reverse transcription kit (Takara Biotechnology Co., Ltd., Dalian, China). Oligo dT and random primers were used to ensure that total mRNA was transcribed to cDNA.

$R T$-qPCR. RT-qPCR was performed using the Mx3005P Multiplex Quantitative PCR system (Stratagene, La Jolla, CA, USA), with qPCR SYBR-Green qPCR reagents (Takara Biotechnology Co., Ltd.). Approximately 100 ng cDNA and a final concentration of $200 \mathrm{nM}$ forward and reverse primer pairs were used for each qPCR reaction, with ROX as the reference dye. The cycling conditions were as follows: an initial cycle at $95^{\circ} \mathrm{C}$ for $15 \mathrm{sec}$, followed by 40 cycles of $95^{\circ} \mathrm{C}$ degeneration for $5 \mathrm{sec}, 60^{\circ} \mathrm{C}$ annealing and extension for $33 \mathrm{sec}$. The melting curve analysis was carried out on each sample to ensure a single amplicon. Results were exported to Microsoft Excel for analysis.

Evaluation of gene stability and statistical analysis. The stability of candidate reference genes was evaluated by various algorithms, including the geNorm (17), NormFinder and BestKeeper programs and the comparative $\Delta \mathrm{Ct}$ method (22).

Normalization of collagen I, collagen II and aggrecan as target genes. The effects of the reference gene variance on the expression of collagen I, collagen II and aggrecan were analyzed with the $2^{-\Delta \Delta \mathrm{Ct}}$ method, as markers of chondrogenic and osteogenic differentiation of BMSCs. The primer pair 


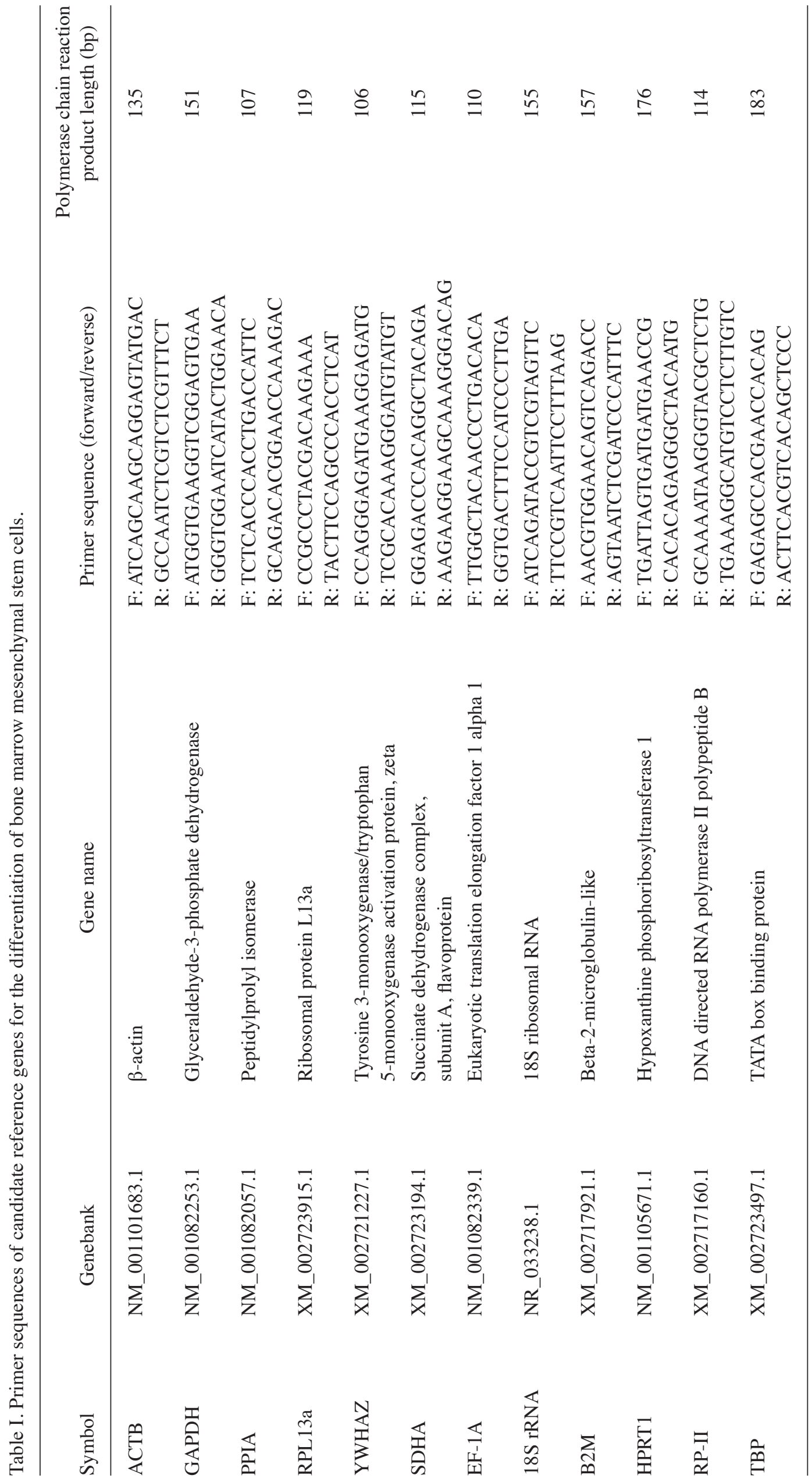


Table II. Primer sequences of target genes collagen II, aggrecan and collagen I as markers of chondrogenic and osteogenic differentiation of bone marrow mesenchymal stem cells.

\begin{tabular}{|c|c|c|c|c|}
\hline Symbol & Genebank & Gene name & Primer sequence (forward/reverse) & $\begin{array}{l}\text { Polymerase chain reaction } \\
\text { product length (bp) }\end{array}$ \\
\hline Collagen II & NM_001195671.1 & Collagen type II & $\begin{array}{l}\text { F: CTGTCCTGTGCGACGACATA } \\
\text { R: TCCTTTCTGCCCCTTTGGTC }\end{array}$ & 140 \\
\hline Aggrecan & NW_003159560.1 & Aggrecan & $\begin{array}{l}\text { F: TGGAGAAGCCCTTGCATCTG } \\
\text { R: TGGGACGGAGGATGCTTCTA }\end{array}$ & 82 \\
\hline Collagen I & AY633663.1 & Collagen type I & $\begin{array}{l}\text { F: GAGGTGGACACCACCCTCAA } \\
\text { R: CCAGTGTCCATGTCGCAGAA }\end{array}$ & 200 \\
\hline
\end{tabular}

sequence target genes are listed in Table II. The expression of genes on day 1 was designated as the calibrator, and the target genes were normalized by the 12 reference genes and the recommended combination of geNorm, Normfinder and BestKeeper.

\section{Results}

Isolation, culture and differentiation of rabbit BMSCs. The techniques for the isolation of rabbit BMSCs have been well investigated and are well established. In accordance with a previous study (21), the rabbit BMSCs were isolated after three passages. The cells are shown in Fig. 1, and morphological analysis clearly suggests highly purified BMSCs.

Expression profiles of candidate reference genes. The $\mathrm{Ct}$ values of 12 candidate reference genes in the chondrogenic and osteogenic differentiation of BMSCs were calculated (Fig. 2) to compare the various gene expression profiles. The $\mathrm{Ct}$ values for the 12 reference genes ranged from 10.18 (18s rRNA) to 28.29 (GAPDH), demonstrating a wide variation. As shown in Fig. 2, 18s rRNA was highly expressed and GAPDH had the lowest transcription value, with $\mathrm{Ct}$ values ranging from 26.77 to 28.29. The individual reference genes had different $\mathrm{Ct}$ value ranges and no single reference gene had a constant expression level in the chondrogenic and osteogenic differentiation of BMSCs.

Osteogenic differentiation. The stability of reference genes during osteogenic differentiation was evaluated by the geNorm, Normfinder and BestKeeper software. As shown in Fig. 3A, geNorm assessed PPIA and HPRT1 as being the most stable genes, followed by the genes TBP, RP-II and EF-1A, while the most unstable genes were B2 M, YWHAZ and $18 \mathrm{~s}$ rRNA. PPIA and HPRT1 were sufficient as reference genes, with the V2/3 value of 0.071 much lower than the suggested cut-off value of 0.15 . Additionally, similar results on gene stability were obtained by Normfinder (Fig. 3B) and BestKeeper (Fig. 3C), which demonstrated that RPL13a was the most stable reference gene, with a stability value of 0.091 and a standard deviation of 0.06 . It was clearly demonstrated that RPL13a, ACTB and EF-1A were moderately stable, while the best combination of two reference genes was 18s rRNA and HPRT1, with a stability value of 0.055 in Normfinder.

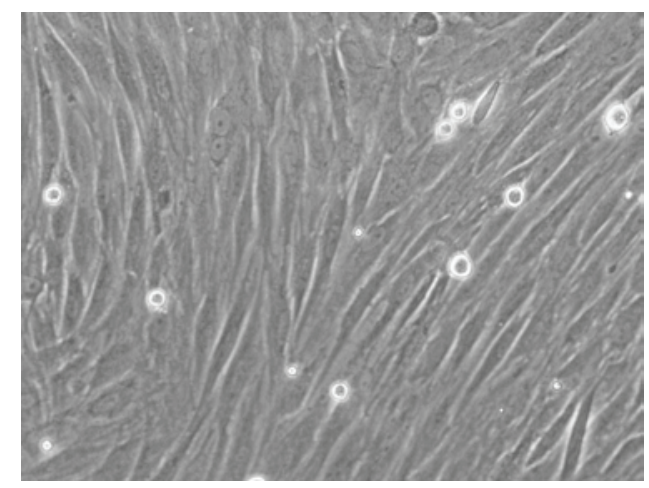

Figure 1. Characterization of isolated marrow stromal cells. Cells were cultured from bone marrow and are shown at 9 days after plating.

Conversely, the stability of B2M, YWHAZ and 18s rRNA was inconsistent with the geNorm results, with higher stability values and standard deviation than the other genes.

Chondrogenic differentiation. The stability of reference genes during chondrogenic differentiation was also evaluated by the geNorm, Normfinder and BestKeeper software. As shown in Fig. 4A, geNorm revealed RPL13 and YWHAZ to be the most stable genes, followed by SDHA, GAPDH and ACTB. The most unstable genes were B2 M, HPRT1 and 18s rRNA, with stability M-values higher than 0.5. Additionally, the V2/3 value was 0.074 , much lower than the suggested cut-off value, demonstrating that RPL13 and YWHAZ were sufficient as reference genes to evaluate the chondrogenic differentiation of BMSCs. Moreover, Normfinder (Fig. 4B) and BestKeeper (Fig. 4C) exhibited similar results on gene stability in the chondrogenic differentiation, which were a little different from the results of geNorm. Normfinder assessed YWHAZ as the most stable gene, followed by GAPDH, PPIA and RPL13. However, the value of the best combination of the two genes PPIA and RPL13 was 0.078, which was much larger than the value for YWHAZ alone, suggesting that YWHAZ alone is sufficient for normalization. Similar results were observed with BestKeeper, demonstrating PPIA, YWHAZ, GAPDH and ACTB to be the most stable genes. Conversely, B2M, HPRT1 and 18s rRNA were revealed to be the most unstable genes during chondrogenic differentiation, with higher M-values, stability values and standard deviation than the other genes. 


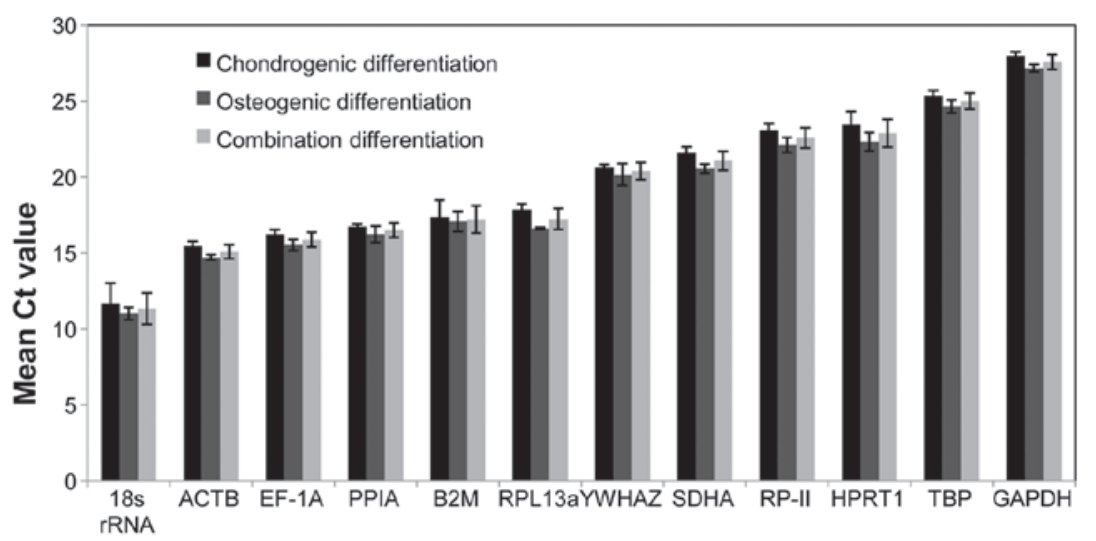

Figure 2. Expression profiles of 12 reference genes, shown as Ct values in chondrogenic and osteogenic differentiation of bone marrow mesenchymal stem cells at 1,7 and 14 days.
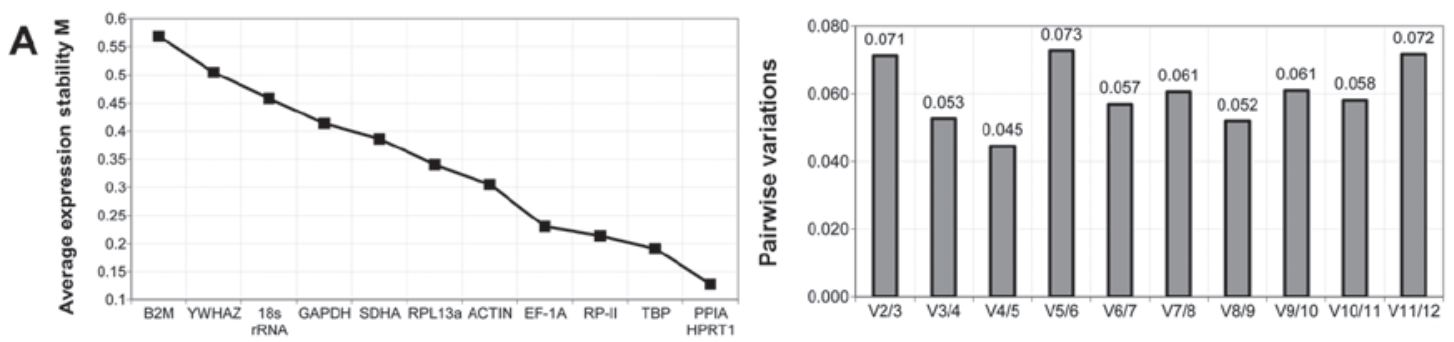

B

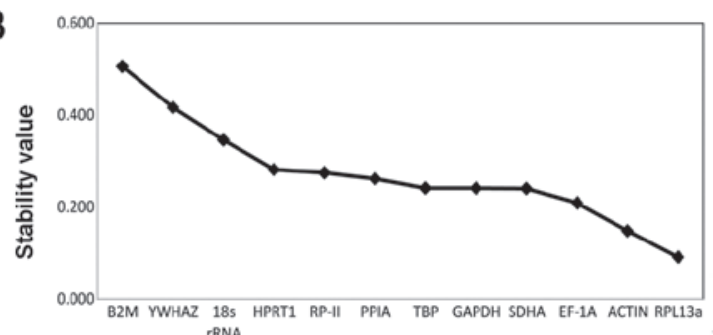

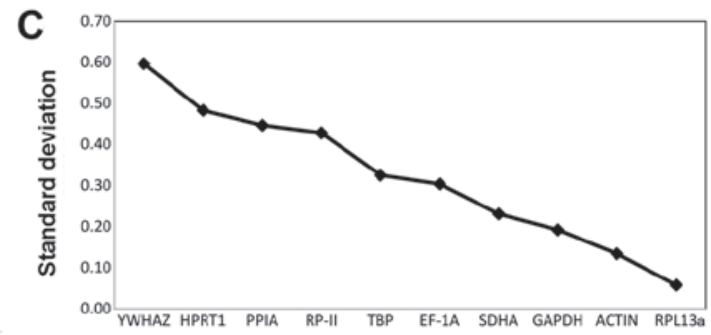

Figure 3. Stability analysis of 12 reference genes in osteogenic differentiation of bone marrow mesenchymal stem cells by geNorm (A), Normfinder (B) and BestKeeper (C). (A) geNorm: Average expression stability measure (M) of reference genes and the optimal reference gene number for normalization. Lower M-values, stability values and standard deviation imply greater stability.

A

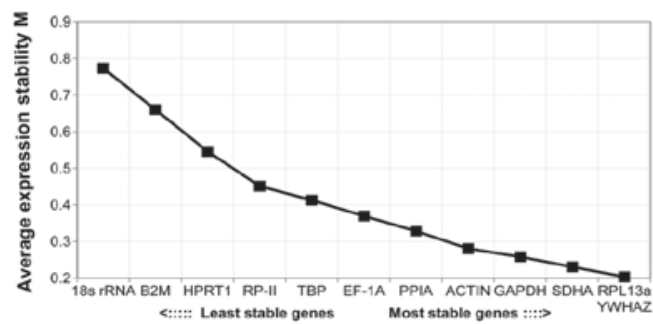

B

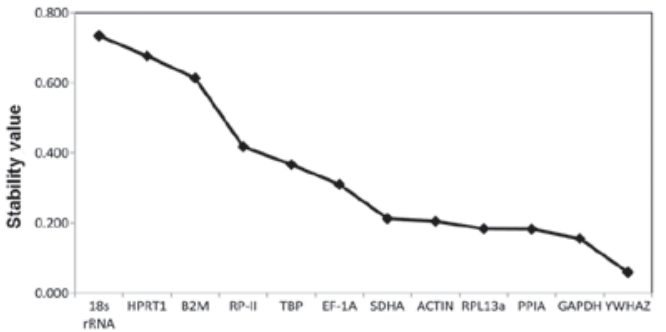

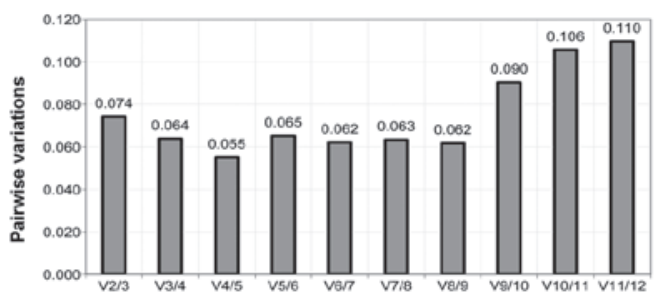

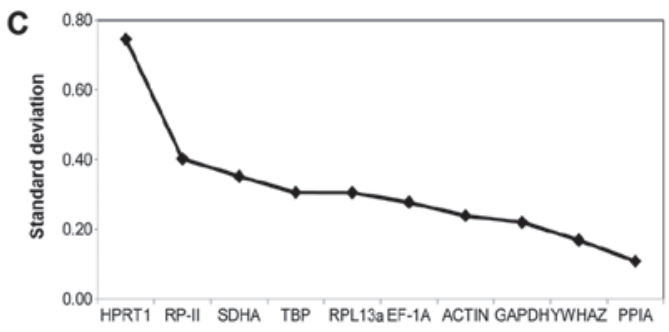

Figure 4. Stability analysis of 12 reference genes in chondrogenic differentiation of bone marrow mesenchymal stem cells by geNorm (A), Normfinder (B) and BestKeeper (C). (A) geNorm: Average expression stability measure (M) of reference genes and the optimal reference gene number for normalization. Lower M-values, stability values and standard deviation imply greater stability. 

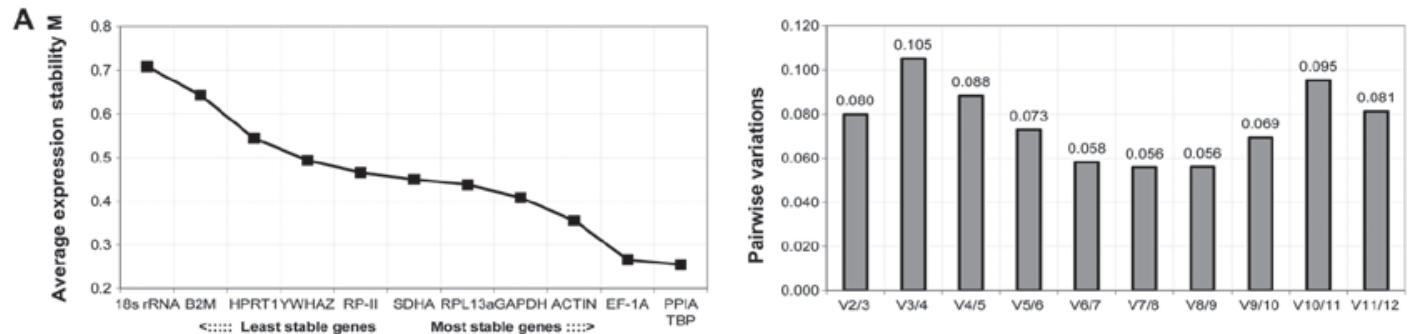

B
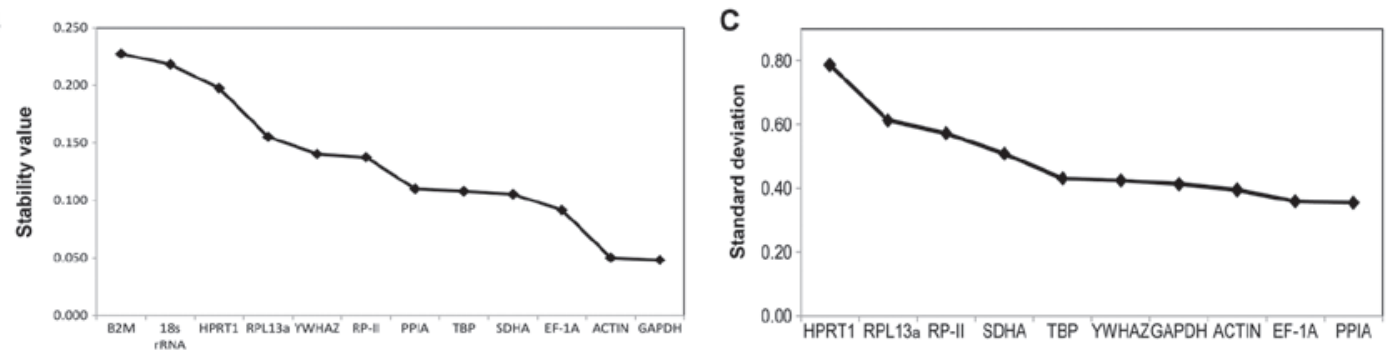

Figure 5. Stability analysis of 12 reference genes in combined osteogenic and chondrogenic differentiation of bone marrow mesenchymal stem cells by geNorm (A), Normfinder (B) and BestKeeper (C). (A) geNorm: Average expression stability measure (M) of reference genes and the optimal reference gene number for normalization. Lower M-values, stability values and standard deviation imply greater stability.

Osteogenic and chondrogenic differentiation. The stability of reference genes during combined osteogenic and chondrogenic differentiation was also evaluated using the geNorm, Normfinder and BestKeeper software. As shown in Fig. 5A, PPIA and TBP were demonstrated to be the most stable genes in the geNorm analysis, followed by EF-1A, ACTB and GAPDH, while the most unstable genes were 18s rRNA, B2 M and HPRT1, with stability M-values higher than 0.5. Additionally, the V2/3 value was 0.080 , much lower than the suggested cut-off value, demonstrating that PPIA and TBP were sufficient as reference genes to evaluate the chondrogenic and osteogenic differentiation of BMSCs. Moreover, the results of BestKeeper (Fig. 5C) were similar to those of geNorm, with PPIA being the most stable gene followed by EF-1A, ACTB and GAPDH. However, little difference existed with Normfinder. It assessed GAPDH as being the most stable gene rather than PPIA, followed by ACTB, EF-1A and SDHA, and the best combination of two genes was ACTB and GAPDH, presenting a higher stability value than the single genes. Conversely, B2M, HPRT1 and 18s rRNA were the most unstable genes during chondrogenic and osteogenic differentiation, with higher M-values, stability values and standard deviation than the other genes, which was inconsistent with the results from geNorm.

Expression profiles of marker genes normalized against various reference genes in the differentiation of BMSCs. The effects of reference gene variance on the expression profiles of the chondrogenic and osteogenic differentiation markers were investigated (Fig. 6). For clarity, the relative expression of the marker genes for chondrogenic and osteogenic differentiation-related genes collagen II, aggrecan and collagen I was obtained with the $2^{-\Delta \Delta \mathrm{Ct}}$ method normalized by the 12 reference genes and the best reference combination recommended by geNorm, Normfinder and BestKeeper. The results revealed that the expression of collagen II and aggrecan increased with time by during the chondrogenic differentiation of BMSCs,
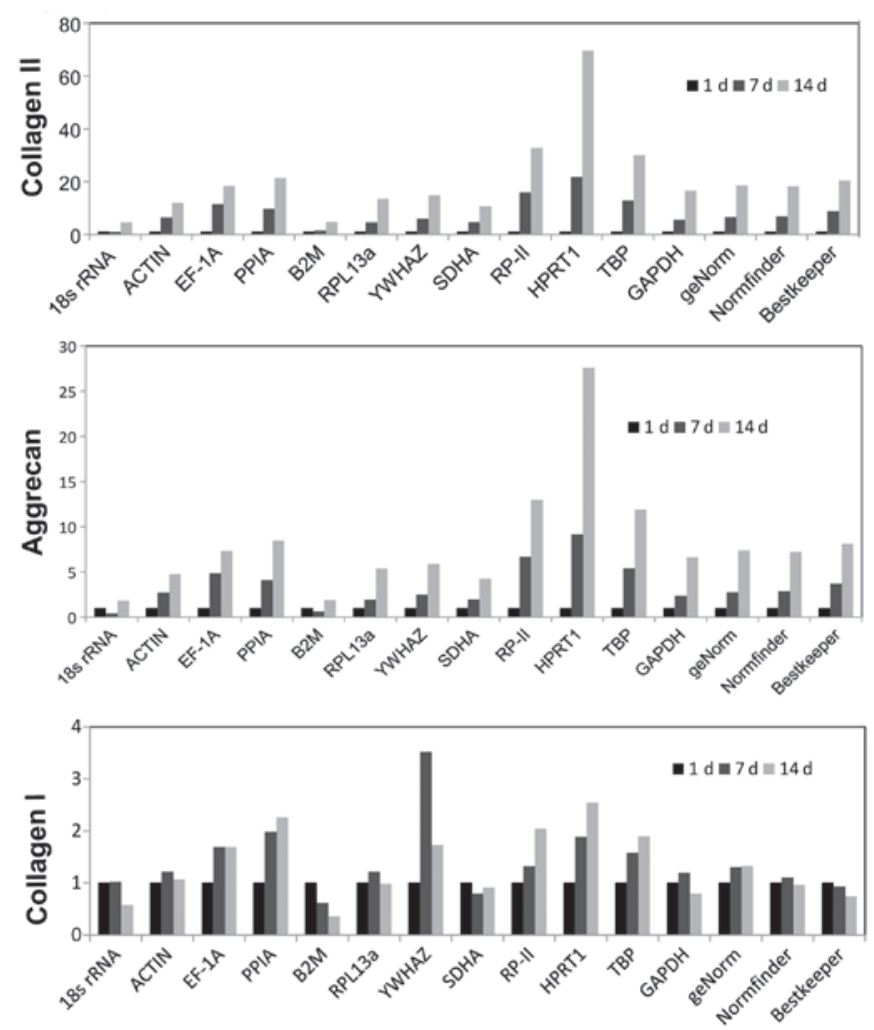

Figure 6. Relative quantities of collagen II, aggrecan and collagen I as chondrogenic and osteogenic differentiation markers of bone marrow mesenchymal stem cells, respectively normalized by various reference genes using the $2^{-\Delta \Delta \mathrm{Ct}}$ method.

while collagen I increased during osteogenic differentiation. As shown in Fig. 6A, the increasing trends of collagen II and aggrecan were almost the same during chondrogenic differentiation when the genes were normalized by the best reference combination recommended by geNorm, Normfinder and BestKeeper. However, the expression trends were quite 
different when the genes were normalized by B2M, HPRT1 and 18s rRNA, the most unstable genes during chondrogenic differentiation. The result is in accordance with the validation of reference genes analysis. Moreover, as for the osteogenic differentiation of BMSCs (Fig. 6B), the increased expression trends were observed when the genes were normalized by PPIA, RP-II, HPRT1, TBP and the combination of PPIA and HPRT1. However, results were different based on other reference genes, demonstrating the significance of suitable reference genes in the analysis of osteogenic differentiation, and the results were consistent with the geNorm analysis.

\section{Discussion}

Bone marrow mesenchymal stem cells are considered as multipotent cells (23), existing in the bone marrow, which represent a multilineage potential to differentiate into mesodermal lineages $(1,2)$. It is the adult bone mesenchymal stem cells that contribute to the regeneration of mesenchymal tissues following trauma or disease or during aging (24-26). Evidence indicates the osteogenic and chondrogenic differentiation ability of BMSCs in vitro and in vivo. BMSCs exhibited a stable phenotype and remained as a monolayer in vitro, maintaining their multilineage differentiation potential (1). Moreover, particular techniques to induce BMSC differentiation into chondrogenic $(8,27)$, osteogenic (9), adipogenic (10) and myogenic cells (11) have been well investigated. Furthermore, implantation of BMSCs to defect organs or tissues has proven successful in a variety of animal models, where the BMSCs differentiated into the appropriate phenotypes according to the local stimulating factors (28). Im et al transported the cultured BMSCs to full-thickness defect rabbit models and achieved a satisfactory result (29). In the study of Fuchs et al, ovine BMSCs were harvested and seeded onto biodegradable scaffolds, then transplanted into the cartilage defects of ewe femoral trochlea. Three months after the transplantation, the cartilage defects were repaired completely, with high proteoglycan and type II collagen content (30). As for bone regeneration, Niemeyer et al accomplished the repair of sheep tibia defects through implantation of ovine BMSCs seeded on mineralized collagen sponges (31). Liu et al demonstrated that a BMSC sheet as a $3 \mathrm{D}$ scaffold material was a promising strategy in healing a large bone defect area in osteoporosis through the implantation of BMSCs to the defect part of the bone (4). In conclusion, BMSCs demonstrate notable qualities as seed cells in cartilage and bone regeneration. Tissue engineering in BMSCs has made great advances; however, the mechanisms of the differentiations of BMSCs are still unclear. Molecular changes in gene expression profiles during BMSC differentiation are crucial to explain the mechanisms of the multipotent differentiation process.

qPCR is a known, reliable, accurate, fast and sensitive method to quantify gene expression and decipher the properties of BMSC multipotent differentiation. However, an appropriate reference gene is required to be constitutively expressed to eliminate the errors caused by the cell number, reverse transcription and amplification efficiency differences (16). However, evidence has revealed that the expression of widely used reference genes, although occasionally constant in certain experimental conditions, varies considerably in BMSC proliferation and differentiation (18). Research has been carried out to attempt to validate an appropriate reference gene in BMSC differentiation (22), although no conclusion has been reached. Amable et al studied the appropriate reference genes for human mesenchymal cells during expansion and differentiation, demonstrating that HPRT1 was the most stably expressed gene for BMSCs, while the most unstable gene was GAPDH (32). Studer et al (20) and Ragni et al (33) established RPL13a as the most stable reference gene during the chondrogenic and osteogenic differentiation of BMSCs. Quiroz et al also validated RPL13a, GAPDH and ACTB as stable reference genes for osteogenic differentiation of BMSCs (34). Curtis et al demonstrated that EF-1a, RPL13a and YWHAZ were the most stable reference genes for MSC differentiation in an animal model of global cerebral ischemia (18). In conclusion, the recommended reference genes varied according to the different studies, and no consensus was reached, which was consistent with our results. In the present study, the suitable reference genes varied due to the differentiation microenvironments. For example, YWHAZ was the most stable reference gene for the chondrogenic differentiation of BMSCs, while RPL13a (Normfinder and BestKeeper), PPIA and HPRT1 (geNorm) acted as the most efficient reference genes. However, 18s rRNA, HPRT1 and B2M were considered to be the most unstable reference genes in both chondrogenic and osteogenic differentiation of BMSCs, which is in accordance with a number of previous studies $(18,22)$.

ACTB and GAPDH, the most popular reference genes in use worldwide, are not the most effective reference genes under all experimental conditions. Foldager et al reported that the variations of ACTB and GAPDH for hypoxia-cultured human chondrocytes were much larger than for other genes (35), underlining their ineffectiveness as reference genes. The same results were obtained in the propagation, differentiation and hypoxic exposure of adipose-derived stem cells, where the expression of GAPDH was downregulated during chondrogenesis and upregulated under hypoxic conditions, leading to erroneous results in the expression of target genes (36). In the present study, ACTB and GAPDH were not the most stable genes in all experimental conditions, when the BMSCs underwent osteogenic differentiation.

In conclusion, our results demonstrated that the expression of commonly used reference genes varies in the chondrogenic and osteogenic differentiation of BMSCs. We identified YWHAZ, PPIA and GAPDH as suitable reference genes for chondrogenic differentiation, while RPL13a allowed an efficient normalization expression value of interest genes for osteogenic differentiation of BMSCs. Taking both chondrogenic and osteogenic differentiation into consideration, GAPDH, ACTB, PPIA and EF-1A were the most stable genes, which was a little different from the results for chondrogenic and osteogenic differentiation alone, indicating that reference gene stability varied according to the different experimental conditions. However, the most unstable reference genes were 18s rRNA, B2 M and HPRT1 in all analyses, and should be avoided in studies on the differentiation of BMSCs. Our results highlight the significance of using appropriate reference genes in RT-qPCR normalization. 


\section{Acknowledgements}

This study was financially supported by the National Natural Science Foundation of China (project 51273081) and Changchun Science and Technology (project 2012092).

\section{References}

1. Pittenger MF, Mackay AM, Beck SC, et al: Multilineage potential of adult human mesenchymal stem cells. Science 284: 143-147, 1999.

2. Jiang Y, Jahagirdar BN, Reinhardt RL, et al: Pluripotency of mesenchymal stem cells derived from adult marrow. Nature 418 41-49, 2002.

3. Sanchez-Ramos J, Song S, Cardozo Pelaez F, et al: Adult bone marrow stromal cells differentiate into neural cells in vitro. Exp Neurol 164: 247-256, 2000.

4. Lin G, Wang G, Liu G, et al: Treatment of type 1 diabetes with adipose tissue-derived stem cells expressing pancreatic duodenal homeobox 1. Stem Cells Dev 18: 1399-1406, 2009.

5. Rosová I, Dao M, Capoccia B, et al: Hypoxic preconditioning results in increased motility and improved therapeutic potential of human mesenchymal stem cells. Stem Cells 26: 2173-2182, 2008.

6. Zhang W, Zhang F, Shi H, et al: Comparisons of rabbit bone marrow mesenchymal stem cell isolation and culture methods in vitro. PloS One 9: e88794, 2014.

7. Lv FJ, Tuan RS, Cheung KM and Leung VY: Concise review: the surface markers and identity of human mesenchymal stem cells Stem Cells 32: 1408-1419, 2014.

8. Jungebluth P, Alici E, Baiguera S, et al: Tracheobronchial transplantation with a stem-cell-seeded bioartificial nanocomposite: a proof-of-concept study. Lancet 378: 1997-2004, 2011.

9. Guan M, Yao W, Liu R, et al: Directing mesenchymal stem cells to bone to augment bone formation and increase bone mass. Nat Med 18: 456-462, 2012.

10. Karagianni M, Brinkmann I, Kinzebach S, et al: A comparative analysis of the adipogenic potential in human mesenchymal stromal cells from cord blood and other sources. Cytotherapy 15 : 76-88, 2013.

11. Salem HK and Thiemermann C: Mesenchymal stromal cells: current understanding and clinical status. Stem Cells 28 585-596, 2010.

12. Gerson SL: Mesenchymal stem cells: no longer second class marrow citizens. Nat Med 5: 262-264, 1999.

13. Cao L, Yang F, Liu G, et al: The promotion of cartilage defect repair using adenovirus mediated Sox9 gene transfer of rabbit bone marrow mesenchymal stem cells. Biomaterials 32: 3910-3920, 2011

14. Grayson WL, Bunnell BA, Martin E, et al: Stromal cells and stem cells in clinical bone regeneration. Nat Rev Endocrinol: Jan 6, 2015 (Epub ahead of print).

15. Horwitz EM, Prockop DJ, Fitzpatrick LA, et al: Transplantability and therapeutic effects of bone marrow-derived mesenchymal cells in children with osteogenesis imperfecta. Nat Med 5: 309-313, 1999

16. Haller F, Kulle B, Schwager S, et al: Equivalence test in quantitative reverse transcription polymerase chain reaction: confirmation of reference genes suitable for normalization. Anal Biochem 335: 1-9, 2004.

17. Vandesompele J, De Preter K, Pattyn F, et al: Accurate normalization of real-time quantitative RT-PCR data by geometric averaging of multiple internal control genes. Genome Biol 3 : RESEARCH0034, 2002.
18. Curtis KM, Gomez LA, Rios C, et al: EF1alpha and RPL13a represent normalization genes suitable for RT-qPCR analysis of bone marrow derived mesenchymal stem cells. BMC Mol Biol 11: 61, 2010

19. Radonić A, Thulke S, Mackay IM, et al: Guideline to reference gene selection for quantitative real-time PCR. Biochem Biophys Res Commun 313: 856-862, 2004.

20. Studer D, Lischer S, Jochum W, et al: Ribosomal protein 113a as a reference gene for human bone marrow-derived mesenchymal stromal cells during expansion, adipo-, chondro- and osteogenesis. Tissue Engineering Part C Methods 18: 761-771, 2012.

21. Haynesworth S, Goshima J, Goldberg V, et al: Characterization of cells with osteogenic potential from human marrow. Bone 13: 81-88, 1992.

22. Silver N, Best S, Jiang J and Thein SL: Selection of housekeeping genes for gene expression studies in human reticulocytes using real-time PCR. BMC Mol Biol 7: 33, 2006.

23. Owen M: Lineage of osteogenic cells and their relationship to the stromal system. In: Bone and Mineral Research. Peck WA (ed). Vol 3. Elsevier, New York, NY, pp1-25, 1985.

24. Bianco P, Riminucci M, Gronthos S and Robey PG: Bone marrow stromal stem cells: nature, biology and potential applications. Stem Cells 19: 180-192, 2001.

25. Jones E and Yang X: Mesenchymal stem cells and bone regeneration: current status. Injury 42: 562-568, 2011.

26. Sethe S, Scutt A and Stolzing A: Aging of mesenchymal stem cells. Ageing Res Rev 5: 91-116, 2006.

27. Winter A, Breit S, Parsch D, et al: Cartilage-like gene expression in differentiated human stem cell spheroids: A comparison of bone marrow-derived and adipose tissue-derived stromal cells. Arthritis Rheum 48: 418-429, 2003.

28. Pittenger MF, Mackay AM, Beck SC, et al: Multilineage potential of adult human mesenchymal stem cells. Science 284: 143-147, 1999.

29. Im GI, Kim DY, Shin JH, et al: Repair of cartilage defect in the rabbit with cultured mesenchymal stem cells from bone marrow. J Bone Joint Surg Br 83: 289-294, 2001.

30. Fuchs JR, Hannouche D, Terada S, et al: Fetal tracheal augmentation with cartilage engineered from bone marrow-derived mesenchymal progenitor cells. J Pediatr Surg 38: 984-987, 2003.

31. Niemeyer P, Fechner K, Milz S, et al: Comparison of mesenchymal stem cells from bone marrow and adipose tissue for bone regeneration in a critical size defect of the sheep tibia and the influence of platelet-rich plasma. Biomaterials 31: 3572-3579, 2010.

32. Amable PR, Teixeira MVT, Carias RBV, et al: Identification of appropriate reference genes for human mesenchymal cells during expansion and differentiation. PloS One 8: e73792, 2013.

33. Ragni E, Viganò M, Rebulla $\mathrm{P}$, et al: What is beyond a qRT-PCR study on mesenchymal stem cell differentiation properties: how to choose the most reliable housekeeping genes. J Cell Mol Med 17: 168-180, 2013.

34. Quiroz FG, Posada OM, Gallego-Perez D, et al: Housekeeping gene stability influences the quantification of osteogenic markers during stem cell differentiation to the osteogenic lineage. Cytotechnology 62: 109-120,2010.

35. Foldager CB, Munir S, Ulrik-Vinther M, et al: Validation of suitable house keeping genes for hypoxia-cultured human chondrocytes. BMC Mol Biol 10: 94, 2009.

36. Fink T, Lund P, Pilgaard L, et al: Instability of standard PCR reference genes in adipose-derived stem cells during propagation, differentiation and hypoxic exposure. BMC Mol Biol 9: 98, 2008. 